\title{
Detection of Organic Vapors by a Polished Single Mode Optical Fiber Sensor
}

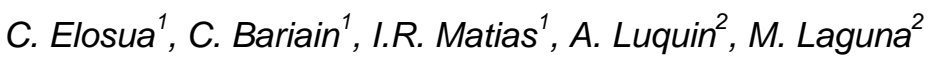 \\ ${ }^{1}$ Public University of Navarre, Campus Arrosadia s/n E31006, Spain \\ ${ }^{2}$ Inst de Sintesis Quimica y Catalisis Homogenea (CSIC), PIz S. Francisco s/n E50006, Spain \\ cesar.elosua@unavarra.es
}

\begin{abstract}
:
Polished Single Mode Fiber (SMF) has been used to develop an evanescent wave sensor whose response varies in presence of Volatile Organic Compounds (VOCs). A vapochromic complex has been fixed along the polished section following the Layer-by-Layer method (LbL). The sensor was studied in a transmission configuration, using a laser @ $1550 \mathrm{~nm}$ as light source and an optical power meter as receptor. The temporal response was registered for different organic vapors, observing distinct dynamic ranges and recovery times: both can be used to discriminate between the VOCs.
\end{abstract}

Key words: Polished fiber, volatile organic compounds, Layer by Layer, evanescent field sensor.

\section{Introduction}

Applications that handle with VOCs have to meet some specific conditions such as a high explosion risk or dangerous toxicity levels. Optical fiber technology offer interesting features to stand these challenges thanks to its passive nature and its remote sensing capability. Therefore, the field of VOCs detection is a potential market niche for optical fiber sensors: as a consequence, efforts are been focused on these devices. Remote sensing and sensors multiplexing features are easier to use with SMF, though it shows a low sensitivity. Nevertheless, polished SMFs make the evanescent field of the signal accessible, enhancing the sensitivity. In this work, a sensor has been developed taking advantage of this cladding elimination, depositing a material sensitive to VOCs.

\section{Experimental}

The polymers used to perform the LbL were Poly Allylamine Hydrochloride (PAH) and Poly Acrylic Acid (PAA); the vapochromic material has the general formula $\left[\mathrm{Au}_{2} \mathrm{Ag}_{2}\left(\mathrm{C}_{6} \mathrm{~F}_{5}\right)_{4}\left(\mathrm{NH}_{3}\right)_{2}\right]_{\text {n }}$. This material suffers reversible changes in its optical properties in presence of VOCs [1]. Once prepared, the sensor is connected between the light source and the optical power meter. It is placed in a stainless chamber where the VOCs are injected and get evaporated.

\section{Sensor Construction}

The fiber is dipped into PAH and PAA solutions, following the LbL procedure. A colloid of the sensing material is prepared with ethanol and a surfactant (sodium dodecyl sulfate, SDS) in order to include the vapochromic complex in the LbL solutions sequence. The construction process is summarized by this expression: $[\mathrm{PAH} / \text { Colloid/PAH/PAA }]_{15}$, representing each stage a immersion in the respective solution [2]. Once the process is over, an homogeneous deposition is fixed onto the fiber, with an average thickness around $1.136 \mu \mathrm{m}$ (Fig. 1).

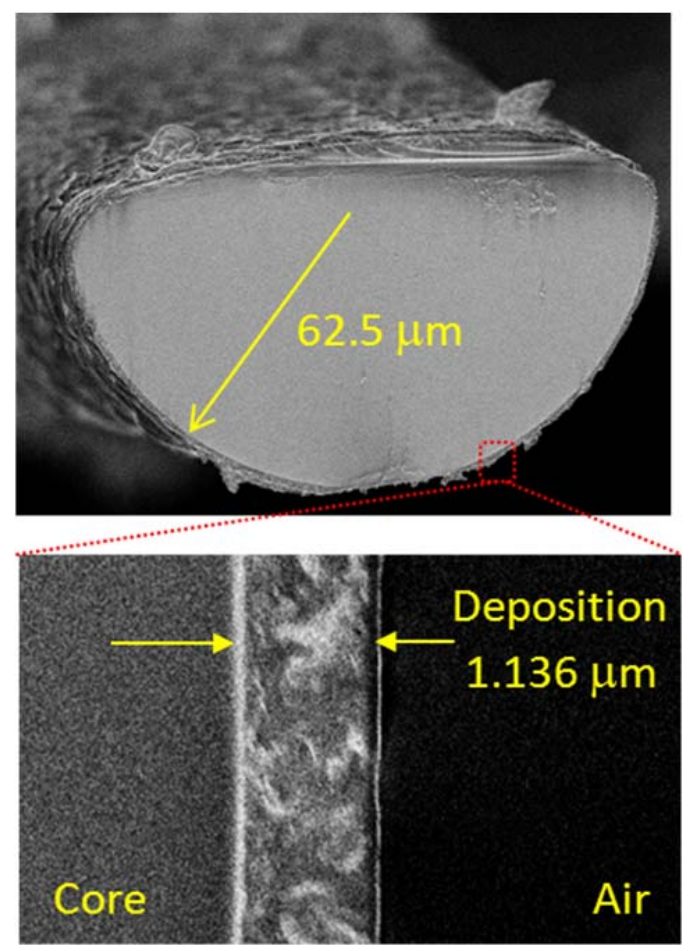

Fig. 1. On the left, an image of the sensor section obtained by a Scanning Electronic Microscope. On the right, an image focused on the core interface. 
It can be observed that the core is almost accessible on the polished side. As a consequence, the evanescent field can be modified depending on the refractive index of the media surrounding the fiber [3]. In the case of the sensing material, it has been demonstrated that its refractive index changes in presence of organic vapors [4], so variations in the signal level should be observed.

\section{Results and Discussion}

The sensor was exposed to saturated atmospheres of different VOCs individually, registering the respective temporal responses. Firstly, three alcohols were used: methanol, ethanol and isopropanol (from highest to lowest polarity). In each case, distinct dynamic range and recovery times were obtained; it is remarkable that for methanol vapors the optical power increases, whereas with the other vapors it get lower. In every case, the initial baseline is recovered (see Fig. 2). Response time is not taken into account because it is affected by the VOC evaporation time.
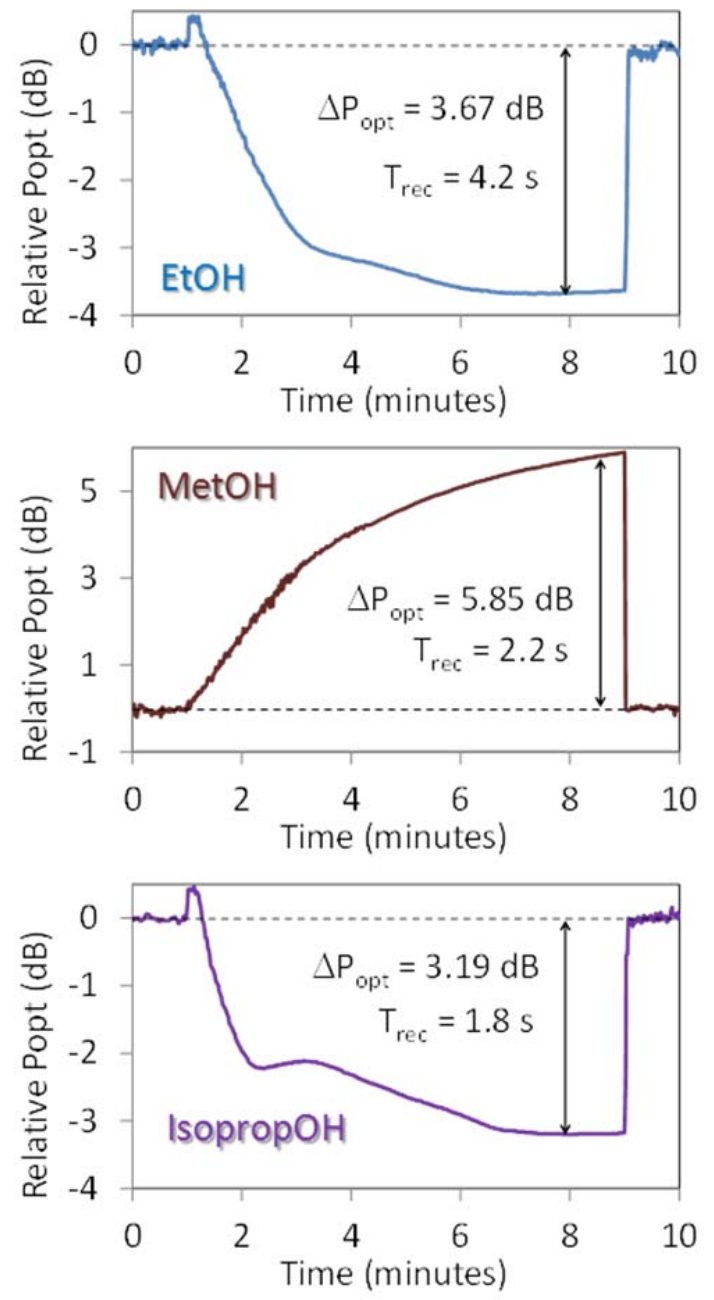

Fig. 2. Temporal responses from the sensor for three different alcohol vapors. Inset, the VOC used, the response dynamic range and the recovery time.
Finally, other non alcoholic VOCs were studied. The shape of the each response is different for every vapor, as well as the dynamic range and the recovery time. Therefore, the sensor can be used to handle with VOCs of different structures.

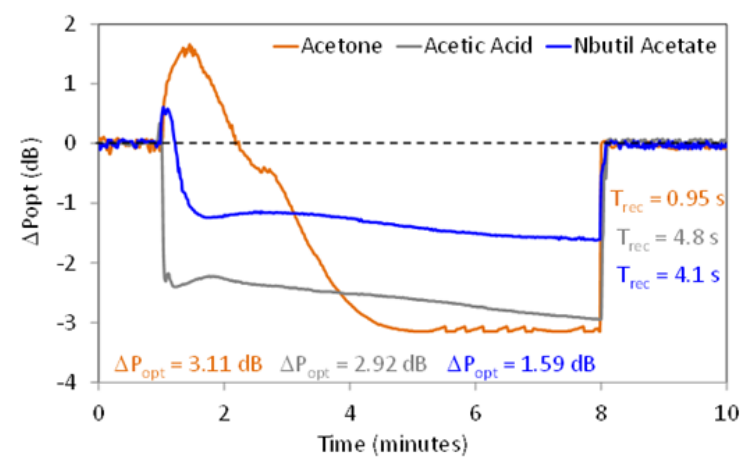

Fig. 2. Temporal responses registered for non alcohol VOCs. Dynamic ranges and recovery times could be used to discriminate between them.

\section{Conclusions}

A transmission sensor has been developed to detect different organic vapors. A polished single mode fiber was used as substrate: therefore, the transduction is based on the evanescent wave modification which is travelling along the polished segment. A nanostructure is deposited on this area, fixing a sensing material whose optical property varies in presence of organic vapors. Specifically, its refractive index varies enough to induce changes in the evanescent field, and so, in the transmitted optical power. The temporal response of the sensor for different alcohols and organic solvents shows that parameters such as the dynamic range or recovery time could be used to identify the different samples.

\section{Acknowledgments}

The authors would like to acknowledge the financial support from the Spanish Ministerio de Educación y Ciencia through projects TEC2010-17805 and TEC2010-20224-C02-01.

\section{References}

[1] R. Uson, A. Laguna, M. Laguna, B.R. Manzano, P.G. Jones, G. M. Sheldrick, Synthesis and reactivity of bimetallic Au-Ag polyfluorophenyl complexes; Crystal and molecular structures of [\{AuAg $\left.\left.\left(\mathrm{C}_{6} \mathrm{~F}_{5}\right)_{2}\left(\mathrm{SC}_{4} \mathrm{H} \quad 8\right)\right\}_{n}\right]$ and $\left[\left\{\mathrm{AuAg}\left(\mathrm{C}_{6} \mathrm{~F}_{5}\right)_{2}\left(\mathrm{C}_{6} \mathrm{H}_{6}\right)\right\}_{n}\right]$, Journal of the Chemical Society, Dalton Transactions 2, 295-292, (1984).

[2] C. Elosua, C. Bariain, A. Luquin, M. Laguna, I.R. Matias, Optimization of single mode fibre sensors to detect organic vapours Sensors and Actuators B 157 (2), 388-394, (2011). 
[3] C. Bariain, I.R. Matıas, C. Fernandez-Valdivielso, F.J. Arregui, M.L. Rodrıguez-Mendez, J.A. de Saja, Optical fiber sensor based on lutetium bisphthalocyanine for the detection of gases using standard telecommunication wavelengths, Sensors and Actuators B: Chemical, 153-158, (2003).

[4] C. Elosua, C. Bariain, I. R. Matias, F. J. Arregui, A. Luquin, M. Laguna, Volatile alcoholic compounds fibre optic nanosensor, Sensors and Actuators B: Chemical, 444-449, (2006). 